\title{
EVALUACIÓN DE LA GESTIÓN DEL MANTENIMIENTO EN HOSPITALES DEL Instituto ECUATORIANo DE SEguridad SOCIAL DE LA ZONA 3 DEL ECUADOR
}

\author{
Assessment of the Maintenance \\ Management in Hospitals of the \\ ECUADORIAN INSTITUTE OF SOCIAL \\ SECURITY OF ZONA 3 OF ECUADOR
}

\author{
Mayra Viscaíno-Cuzco ${ }^{1, *}$, Sergio Villacrés-Parra ${ }^{1}$, \\ César Gallegos-Londoño ${ }^{1}$ Hernán Negrete-Costales ${ }^{2}$
}

\section{Resumen}

Los fallos en la infraestructura hospitalaria tienen consecuencias inestimables debido a que involucra la vida humana, por lo que los riesgos potenciales que contribuyen al fallo de equipos médicos e infraestructura hospitalaria, tienen que ser identificados, reducidos o eliminados; para ello el mantenimiento y su gestión es una herramienta que se enfoca en asegurar el funcionamiento de un equipo. El propósito de este trabajo fue el obtener una valoración cuantitativa de la gestión del mantenimiento en los hospitales del Instituto Ecuatoriano de Seguridad Social de la Zona 3 del Ecuador. La metodología empleada consta de cinco fases, empezando por la selección de criterios de evaluación, ponderación de criterios, desarrollo del instrumento de evaluación, la validación del instrumento aplicándolo a cuatro hospitales de la Zona 3 del Ecuador; finalmente, la identificación de aspectos con bajo desempeño. Los resultados muestran que la gestión de mantenimiento de los hospitales de la Zona 3 , alcanzaron una valoración cuantitativa promedio de $55,5 / 100$ puntos.

\section{Abstract}

The hospital infrastructure failures have an inestimable impact because they put human lives at stake. Therefore, potential risks contributing to the medical equipment and hospital infrastructure failure must be identified, reduced or eliminated. The maintenance and its management constitute a tool that ensures the equipment performance. The aim of this work was to obtain quantitative data of the maintenance management at the hospitals of Zone 3 of the Ecuadorian public health system. The methodology consists of five phases: assessment criteria selection, criteria weighting, assessment instrument development, validation of the instrument by applying it to the four hospitals of the zone 3 in Ecuador, and identification of low-performing aspects. The results demonstrate that the maintenance management of the hospitals in Zone 3 reached a quantitative average value of $55,5 / 100$ points.

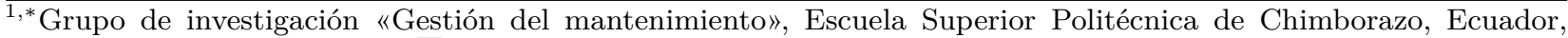
Autor para correspondencia mayra.viscaino@espoch.edu.ec, mayraviscaino@hotmail.com

(D) http://orcid.org/0000-0003-4987-7797, (D) http://orcid.org/0000-0002-9497-9795.

(D) http://orcid.org/0000-0002-8685-7501.

${ }^{2}$ Facultad de Mecánica. Escuela Superior Politécnica de Chimborazo, Ecuador

(D) http://orcid.org/0000-0002-2678-761X.
}

Recibido: 04-05-2019, aprobado tras revisión: 18-06-2019

Forma sugerida de citación: Viscaíno-Cuzco, M.; Villacrés-Parra, S.; Gallegos-Londoño, C. y Negrete-Costales, H. (2019). «Evaluación de la gestión del mantenimiento en hospitales del Instituto Ecuatoriano de Seguridad Social de la Zona 3 del Ecuador». IngEnIUs. N. ${ }^{\circ}$ 22, (julio-diciembre). pp. 59-71. DOI: https://doi.org/10.17163/ings.n22.2019.06. 
En tres hospitales se evidencian debilidades estructurales que comprometen el logro de los objetivos del departamento de mantenimiento, pero existen procesos viables que pueden ser implantados para superar las deficiencias e incrementar el nivel de cumplimiento de las exigencias. Se concluye que la planificación, programación y control del mantenimiento es el criterio con más potencial para mejorar.

Palabras clave: mantenimiento hospitalario, gestión del mantenimiento, proceso analítico jerárquico, evaluación del mantenimiento, sistema de salud público
There are structural deficiencies comprising the achievement of the maintenance department goals in three hospitals, yet there are doable processes that can be implemented to overcome deficiencies and increase the level of requirements fulfillment. We conclude that the criterion of maintenance planning, programming is highly likely to improve.

Keywords: hospital maintenance, maintenance management, analytic hierarchy process, maintenance assessment, public health system. 


\section{Introducción}

En el área industrial se exige un alto desempeño de las máquinas para evitar pérdidas económicas; sin embargo, si se presentan fallos en la infraestructura hospitalaria pueden acarrear consecuencias inestimables porque involucra la vida humana. Un hospital es una estructura compleja compuesta por equipos médicos, industriales y una infraestructura multiforme, que sustenta la prestación de los servicios de salud y por su importancia se requiere que los equipos médicos no presenten fallos imprevistos en su funcionamiento, que la infraestructura proporcione un entorno salubre y seguro como recurso indispensable con el que cuentan los médicos para asegurar una buena atención a los pacientes, generar diagnósticos correctos e inclusive salvaguardar la vida de los mismos.

Esto significa que los riesgos potenciales que contribuyen al fallo de equipos médicos, industriales e infraestructura tienen que ser identificados y reducidos o en lo posible eliminados [1]. En este sentido, el departamento responsable cuenta con el mantenimiento y su gestión como una herramienta que se enfoca esencialmente en asegurar el funcionamiento de un equipo [2] sea médico o industrial. Según Gonnelli et al. [3] el mantenimiento es una parte trascendental del ciclo de vida de un equipo médico; no obstante, la importancia del mantenimiento para la tecnología médica no es tan conocida y se considera solo como una actividad de soporte [4].

En Ecuador el sistema de salud está constituido principalmente por establecimientos públicos y privados [5], siendo el sistema público el que más entidades de salud tiene (81,5\%) [6] y el Instituto Ecuatoriano de Seguridad Social (IESS) que atiende a su población afiliada desde 1970 es una de las instituciones que lo conforman. Un principio rector del sistema de seguridad social ecuatoriano es que funcionará sobre la base de criterios de sostenibilidad, eficiencia, celeridad y transparencia [7]. Entiéndase como eficiencia según la ley de seguridad social «a la mejor utilización económica de las contribuciones y demás recursos del seguro general obligatorio, para garantizar la entrega oportuna de prestaciones suficientes a sus beneficiarios» $[8]$.

Sin embargo, los problemas económicos que enfrenta esta institución desde hace varios años se hacen evidentes en el tipo de atención a sus usuarios; el accionar en atención de salud es crítica e ineficiente según lo expresado por el presidente del Consejo Directivo del IESS [9]. A lo citado se suman problemas administrativos con la infraestructura hospitalaria por falta de mantenimiento; en noviembre de 2018, las autoridades del hospital del IESS de Cotopaxi manifestaron que se suspenderían las cirugías en ese establecimiento de salud porque los equipos de esterilización no estaban disponibles por falta de mantenimiento [10].
La disponibilidad de equipos médicos especializados desempeña un papel fundamental en la prestación de los servicios de salud; mejorar el mantenimiento del equipo médico en términos de efectividad, confiabilidad y disponibilidad significa en última instancia, mejorar la seguridad del paciente y del usuario [3].

De acuerdo con la investigación realizada por Mwanza y Mbohwa, los principales problemas detectados en un hospital que atañen al mantenimiento son una alta indisponibilidad de los equipos y la no ejecución del mantenimiento de equipos según el programa establecido [11]. Debido a que la calidad del mantenimiento es concluyente para brindar una buena atención médica a los pacientes [12] es necesario evaluar su desempeño.

Gonnelli et al. [3] y Orozco et al. [13], indican que medir el desempeño del mantenimiento es un proceso multidisciplinario y se considera importante para una organización porque es una herramienta que permite tomar decisiones gerenciales de manera oportuna. Su objetivo tiende a ser mal interpretado, el propósito no es juzgar a los responsables o cuestionar los resultados de su trabajo, mucho menos sancionarlos, sino identificar aquellos aspectos susceptibles de optimización [14], justificar la inversión hecha en mantenimiento [15] y evitar que los recursos económicos se desperdicien por un mantenimiento deficiente o ineficaz [4].

La evaluación sistemática permite valorar el comportamiento del desempeño de una organización en cualquier área y proponer cambios organizativos y de gestión para lograr una mejora del sistema. Sea que los resultados de la evaluación sean negativos o positivos, las estrategias que se propongan deben apuntar a mejorar continuamente para que la organización funcione con éxito.

Desde el 2014 en el Ecuador se realiza el proceso de acreditación internacional denominada «Accreditation Canada International» (ACI). Cuarenta y cuatro hospitales públicos fueron evaluados [16] con el propósito de obtener una certificación que avale el cumplimiento de estándares de calidad y mejorar la atención en los diferentes centros de salud. En la lista de hospitales evaluados según las prácticas organizacionales requeridas por ACI, el hospital de Pastaza presenta el menor desempeño [17]. Este hospital pertenece a la zona de planificación 3 del Ecuador formada por las provincias de Cotopaxi, Tungurahua, Chimborazo y Pastaza [18].

Se conoce que veintiún hospitales del IESS han sido evaluados hasta el 2018 por ACI [17], organismo que emplea un instrumento mediante el cual valora algunos aspectos de la gestión del mantenimiento de hospitales [19]. Esto se considera positivo, porque el mantenimiento y su gestión constituyen una herramienta que permite alcanzar los objetivos organizacionales y proporcionan una contribución esencial a la seguridad de funcionamiento de un elemento $[2,20]$, en este caso 
tanto de equipos hospitalarios y toda la infraestructura que se requiere para el buen funcionamiento de un hospital.

Para elaborar un instrumento de evaluación es necesario determinar una estructura de criterios de evaluación que permitan valorar efectivamente el desempeño. Aquel es un requisito usado como referencia para comparar la evidencia encontrada, mientras que una evidencia es la información que permite verificar y avalar el cumplimiento de un criterio [21].

Se ha encontrado que en algunos casos se requiere desglosar a los criterios en subcriterios de evaluación $[22,23]$; estos constituyen reglas de valoración del cumplimiento de las exigencias [24] establecidas en el instrumento de evaluación y contribuyen a la valoración de un criterio.

$\mathrm{Al}$ evaluar la gestión del mantenimiento de un hospital se determinan las áreas funcionales que articulan la gestión del mantenimiento como: organización general del mantenimiento, recursos humanos [11, 25], control económico, planificación, programación y control del mantenimiento, tercerización [22], inventario de equipos y su criticidad [26], documentos de mantenimiento, disponibilidad de repuestos en el departamento [11].

Por otra parte, el sistema de acreditación canadiense en lo relacionado con el mantenimiento verifica el cumplimiento de la existencia de un programa implementado de mantenimiento preventivo para todos los dispositivos, equipos y tecnología médica [19], la generación de reportes documentados de mantenimiento preventivo, la aplicación de un proceso para evaluar la efectividad del programa de mantenimiento preventivo, el seguimiento documentado de la investigación de incidentes y problemas que involucran los dispositivos, equipos y la tecnología médica, apuntando a identificar las causas de los fallos. Según lo mencionado, el propósito de este trabajo es diseñar un método que permita la valoración cuantitativa de la gestión del mantenimiento hospitalario, a través de responder a la pregunta de investigación: ¿cómo se puede evaluar la gestión del mantenimiento en los hospitales?

Conocer una valoración cuantitativa de los criterios y subcriterios evaluados permitirá identificar puntualmente oportunidades de mejora en el área de mantenimiento de los hospitales; una metodología que permite lograr este objetivo se basa en [27]: la selección y ponderación de los criterios y subcriterios de evaluación empleando la herramienta Proceso Analítico Jerárquico (PAJ o AHP) [20] y empleada para priorizar cuantitativamente criterios de evaluación [23, 26, 27]; luego el desarrollo de un instrumento de evaluación, la validación del método a través de la evaluación en sitio a las instituciones de salud y, finalmente, la identificación de criterios con menor desempeño.

\section{Materiales y métodos}

Para desarrollar este trabajo se empleó una metodología cualitativa, el proceso metodológico se estructura en cinco fases [27]:

1. Selección de criterios y subcriterios para la evaluación de la gestión del mantenimiento de instalaciones hospitalarias

2. Ponderación de los criterios y subcriterios de evaluación

3. Desarrollo del instrumento de evaluación

4. Validación del método, a través de la evaluación de la gestión de mantenimiento de los cuatro establecimientos hospitalarios de la Zona 3

5. Identificación de aspectos con bajo desempeño

\subsection{Primera fase}

De la revisión de la literatura se recopilaron criterios y subcriterios de evaluación y para su selección se consultó a jefes de mantenimiento hospitalario de la zona, docentes de la carrera de Ingeniería de Mantenimiento de la Escuela Superior Politécnica de Chimborazo (ESPOCH) y profesionales de cuarto nivel en gestión del mantenimiento, quienes valoraron si los criterios y subcriterios se adaptan a las exigencias y contexto del área de estudio, identificándose y seleccionándose siete criterios y veinte subcriterios; además, se estableció el objetivo de cada subcriterio de la siguiente manera:

\subsubsection{CS: Contratación de servicios de mantenimiento}

CS1 - Política de contratación: evaluar si existen definidos los criterios bajo los cuales se determinará al mejor contratista, quien estará a cargo de realizar los trabajos tanto de mantenimiento preventivo y correctivo, considerando la experiencia del contratista en años y en número de contratos de servicios de mantenimiento hospitalario que haya ejecutado.

CS2 - Supervisión de los trabajos de mantenimiento: verificar si el personal técnico del hospital supervisa que el contratista cumpla con los lineamientos y criterios establecidos en el contrato de servicios de mantenimiento.

CS3 - Especificaciones técnicas: garantizar que todas las actividades de mantenimiento contratadas se ejecuten utilizando materiales y equipos que cumplan las especificaciones establecidas, que el personal que interviene tenga la experticia estipulada y que se siga la metodología definida en la oferta del servicio. 


\subsubsection{RH: Recursos humanos}

RH1 - Capacitación y entrenamiento: valorar si se han definido los requerimientos de capacitación que necesite el personal de mantenimiento de equipos e infraestructura hospitalaria para el cumplimiento de sus actividades, y si se ha establecido un plan de capacitación que permita dar seguimiento al porcentaje de cumplimiento del plan.

RH2 - Formación profesional: determinar si el personal responsable del departamento o área de mantenimiento de equipos médicos e infraestructura hospitalaria tiene formación profesional de tercer o cuarto nivel a fin al cargo que desempeña.

RH3 - Cantidad de personal de mantenimiento: evaluar en función del número de órdenes de trabajo de mantenimiento retrasadas, si existe el suficiente número de técnicos para realizar las actividades de mantenimiento preventivo y correctivo que aseguren la disponibilidad tanto de infraestructura y de equipo hospitalario.

\subsubsection{MI: Manejo de inventarios de bodega para mantenimiento}

MI1 - Maestro de ítems: verificar que la bodega de repuestos y materiales para mantenimiento disponga de un maestro de ítems y evaluar según la opinión de los técnicos de mantenimiento qué porcentaje del maestro de ítems tiene errores en la descripción de los ítems o si existe duplicidad de ítems o si las unidades de despacho están mal asignadas.

MI2 - Inventario valorado de ítems: constatar que los repuestos y los materiales para mantenimiento estén correctamente valorados incluyendo los que se encuentren en las sub-bodegas.

MI3 - Control de existencias: constatar que se tiene un control de las existencias de los repuestos y materiales para mantenimiento tanto en la bodega principal como en las sub-bodegas.

\subsubsection{PP: Planificación programación y control}

PP1 - Indicadores de mantenimiento: determinar cuántos indicadores de gestión de mantenimiento se han puesto en marcha en el hospital.

PP2 - Plan de mantenimiento: evaluar si se posee un plan de mantenimiento preventivo y el nivel de planificación logístico de las tareas de mantenimiento que lo componen.

PP3 - Programación de actividades de mantenimiento: establecer el nivel de planificación de soporte logístico en el proceso de programación de las órdenes de trabajo de mantenimiento preventivo y correctivo.

PP4 - Análisis de criticidad basado en riesgo: valorar la complejidad de la metodología que el hospital empleó para establecer la criticidad de los equipos e infraestructura hospitalaria.

PP5 - Inventario de bienes a mantener: evaluar el porcentaje de los equipos e infraestructura hospitalaria que se encuentra codificada e inventariada y constatar si el código se encuentra ubicado físicamente en la infraestructura y en cada equipo que requiere mantenimiento.

PP6 - Documentos de mantenimiento: determinar si el hospital posee los documentos mínimos de mantenimiento como orden de trabajo y requisición de materiales. Y si la información registrada en estos, es la base para la elaboración de los indicadores de mantenimiento.

\subsubsection{OM: Organización del mantenimiento}

OM1 - Políticas del mantenimiento: valorar si el hospital posee políticas de mantenimiento, si estas están actualizadas y si han sido socializadas con el personal del hospital.

OM2 - Herramienta informática para la gestión de mantenimiento: establecer si el hospital posee una herramienta informática tipo GMAO (Gestión de mantenimiento asistido por ordenador), CMMS (computerized maintenance management system) o EAM (enterprise asset management) para gestionar la información de la gestión del mantenimiento y el grado de utilización de la misma.

\subsubsection{CE: Control económico}

CE1 - Presupuesto de mantenimiento: determinar si la metodología empleada para elaborar los presupuestos tanto de mantenimiento preventivo y correctivo de los equipos e infraestructura hospitalaria es técnica y detallada.

CE2 - Porcentaje de cumplimiento de presupuesto ejecutado: evaluar el porcentaje de cumplimiento del presupuesto de mantenimiento del año anterior.

\subsubsection{MC: Mantenimiento correctivo}

MC1 - Documentación y análisis de fallos, consecuencias y efectos: evaluar si se registra información importante sobre los fallos que sufren los equipos e infraestructura hospitalaria y si se aplican metodologías de análisis para disminuir su probabilidad de ocurrencia.

\subsection{Segunda fase}

Para la determinación de las ponderaciones de los criterios y subcriterios de evaluación se aplicó el método proceso AHP, desarrollado por Saaty [28]; usado en investigaciones que tienen por objetivo la priorización 
de alternativas $[26,29,30]$ y ha sido empleado en estudios relacionados con el mantenimiento [23,31]. El procedimiento se puede sintetizar en cuatro pasos [28]:

\subsubsection{Especificación del problema de decisión multicriterio}

La estructura jerárquica consta de tres niveles (Figura 1):

Nivel 1: indica el objetivo de la aplicación de la técnica AHP, en este caso es ponderar los criterios y subcriterios de evaluación.

Nivel 2: consta de los siete criterios que se van a considerar en la evaluación de la gestión del mantenimiento.

Nivel 3: conformado por veinte subcriterios que contribuyen a la valoración de los criterios de evaluación.

\subsubsection{Construcción de las matrices de com- paración pareada}

Para la aplicación del método AHP se realizaron consultas a especialistas relacionados con el área en estudio, se consideraron tres grupos de interés relacionados con el mantenimiento y su gestión. El grupo de treinta especialistas lo conformaron diez jefes de mantenimiento de hospitales de la zona, diez expertos de mantenimiento con grado académico de cuarto nivel en el área, diez docentes de la carrera de Ingeniería de Mantenimiento de la ESPOCH; en promedio la experiencia en el área de mantenimiento de los decisores es de seis años, quienes valoraron las alternativas comparándolas una con otra según la escala de la Tabla 1 del método AHP.

Tabla 1. Escala fundamental de comparación [32]

\begin{tabular}{|c|c|c|}
\hline Valor & Definición & Comentarios \\
\hline 1 & $\begin{array}{c}\text { Igual } \\
\text { importancia }\end{array}$ & $\begin{array}{c}\text { El criterio A es igual de } \\
\text { importante que el criterio B }\end{array}$ \\
\hline 3 & $\begin{array}{c}\text { Importancia } \\
\text { moderada }\end{array}$ & $\begin{array}{c}\text { La importancia y el juicio } \\
\text { favorecen ligeramente al } \\
\text { criterio A sobre el B }\end{array}$ \\
\hline 5 & $\begin{array}{l}\text { Importancia } \\
\text { grande }\end{array}$ & $\begin{array}{l}\text { La experiencia y el juicio } \\
\text { favorecen fuertemente al } \\
\text { criterio A sobre el B }\end{array}$ \\
\hline 7 & $\begin{array}{l}\text { Importancia } \\
\text { muy grande }\end{array}$ & $\begin{array}{l}\text { El criterio A es mucho más } \\
\text { importante que el B }\end{array}$ \\
\hline 9 & $\begin{array}{l}\text { Importancia } \\
\text { extrema }\end{array}$ & $\begin{array}{l}\text { La mayor importancia del } \\
\text { criterio A sobre el B está } \\
\text { fuera de toda duda }\end{array}$ \\
\hline $2,4,6$ y 8 & \multirow{2}{*}{\multicolumn{2}{|c|}{$\begin{array}{l}\text { Valores intermedios entre los anteriores, } \\
\text { cuando es necesario matizar } \\
\text { Si el criterio A es de importancia grande } \\
\text { frente al criterio B, las notaciones } \\
\text { serían las siguientes: } \\
\text { Criterio A frente a criterio B: } 5 / 1 \\
\text { Criterio B frente a criterio A: } 1 / 5\end{array}$}} \\
\hline $\begin{array}{l}\text { Recíprocos } \\
\text { de lo } \\
\text { anterior }\end{array}$ & & \\
\hline
\end{tabular}

Con la valoración de los decisores se construyeron las matrices de comparación pareada (por cada decisor) para los criterios y subcriterios de evaluación respectivamente, una matriz de comparación pareada es una matriz cuadrada Anxn, donde n es el número de criterios o subcriterios, según corresponda y se construyeron según lo descrito en las Ecuaciones 1 y 2.

$$
\begin{gathered}
A_{n x n}=\left[\begin{array}{cccc}
1 & a_{12} & \cdots & a_{1 n} \\
a_{21} & 1 & \cdots & a_{2 n} \\
\vdots & \vdots & \ddots & \vdots \\
a_{n 1} & a_{n 2} & \cdots & 1
\end{array}\right] \\
A_{n x n}=\left[\begin{array}{cccc}
1 & a_{12} & \cdots & a_{1 n} \\
1 / a_{21} & 1 & \cdots & a_{2 n} \\
\vdots & \vdots & \ddots & \vdots \\
1 / a_{n 1} & 1 / a_{n 2} & \cdots & 1
\end{array}\right]
\end{gathered}
$$

\subsubsection{Cálculo del ratio de consistencia (CR)}

Permite determinar si existen contradicciones en la valoración del decisor, este índice resulta del cociente entre el índice de consistencia (IC) y el índice de consistencia aleatoria (ICA) aplicando la Ecuación 3.

$$
C R=\frac{I C}{I C A}
$$

Los valores del índice de consistencia aleatorio son establecidos por el método AHP y están en función del tamaño de la matriz (Tabla 2).

Tabla 2. Índices de consistencia aleatoria [32]

\begin{tabular}{cc}
\hline $\begin{array}{c}\text { Tamaño de } \\
\text { la matriz (n) }\end{array}$ & $\begin{array}{c}\text { Índice de consistencia } \\
\text { aleatoria (ICA) }\end{array}$ \\
\hline 1 & 0 \\
2 & 0 \\
3 & 0,52 \\
4 & 0,89 \\
5 & 1,11 \\
6 & 1,25 \\
7 & 1,35 \\
8 & 1,4 \\
9 & 1,45 \\
10 & 1,49 \\
\hline
\end{tabular}

Para el cálculo del IC, se necesita conocer el valor de $\lambda_{\text {máx }}$ y para obtenerlo primero se normalizó la matriz A mediante la suma, obteniéndose la matriz de comparación pareada normalizada $A_{\text {norm }}$, aplicando la Ecuación 4 da como resultado la Ecuación 5. 
Viscaíno-Cuzco et al. / Evaluación de la gestión del mantenimiento en hospitales del Instituto Ecuatoriano de

A partir de la matriz $A_{\text {norm }}$, se obtiene el vector $\lambda_{n o r m}=\left[\begin{array}{cccc}\frac{a_{11}}{\sum_{n=1}^{n} a_{n 1}}+ & \frac{a_{12}}{\sum_{n=1}^{n} a_{n 2}}+\cdots+ & \frac{a_{1 n}}{a_{n=1}^{n} a_{n n}} \\ \frac{\sum_{21}^{n} a_{n 1}}{n}+ & \sum_{n=1}^{n} a_{n 2} & \cdots+ & \sum_{n=1}^{n} a_{2 n} \\ \vdots & \vdots & & \vdots \\ \frac{a_{n 1}}{a_{n=1}^{n} a_{n 1}}+ & \frac{a_{n 2}}{\sum_{n=1}^{n} a_{n 2}}+ & \cdots+ & \frac{a_{2 n}}{\sum_{n=1}^{n} a_{n n}}\end{array}\right]$ de prioridades globales B (Ecuación 6).

$(4)$

$$
A_{\text {norm }}=\left[\begin{array}{c}
b_{1} \\
1 \\
b_{2} \\
\vdots \\
\vdots \\
b_{n}
\end{array}\right]
$$

$$
B=\left[\frac{b_{1}}{n}, \quad \frac{b_{2}}{n}, \quad \cdots \quad \frac{b_{n}}{n},\right]
$$

Luego, se calcula del vector fila total $\mathrm{C}$ que se obtiene del producto de matrices $\mathrm{A} \times \mathrm{B}$ (Ecuación 7).

$$
A \times B=C=\left[\begin{array}{llll}
c_{1}, & c_{2} & \cdots & c_{n}
\end{array}\right]
$$

Se realiza el cociente entre los elementos correspon(5) dientes de las matrices $\mathrm{C}$ y B, obteniéndose un vector columna D, al promediar sus elementos proporciona el valor de $\lambda_{\text {máx }}$ con el cual se calcula el índice de consistencia empleando la Ecuación 8.

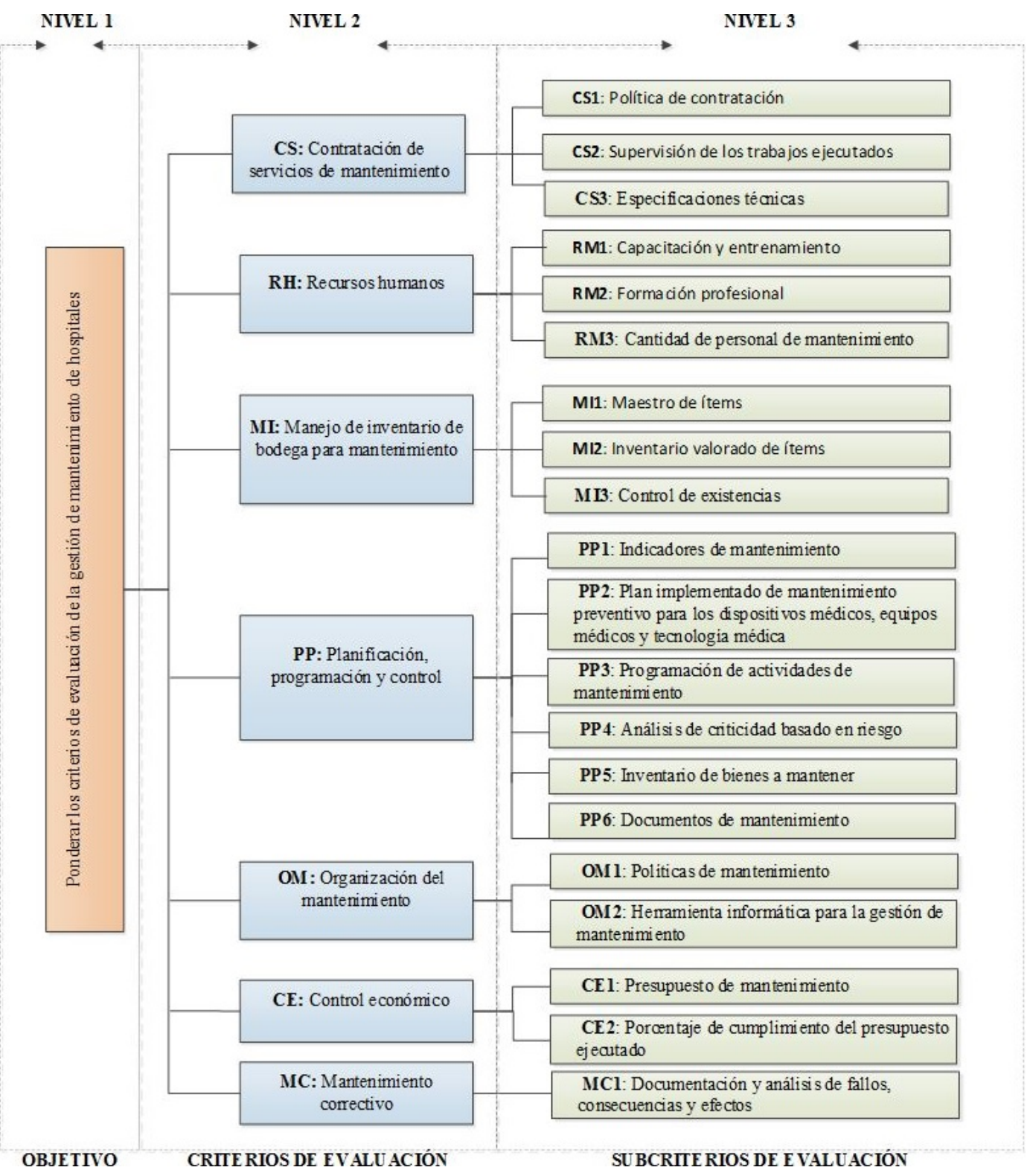

Figura 1. Estructura jerárquica del problema de priorización. 


$$
I C=\frac{\lambda_{m a ́ x-n}}{n-1}
$$

Conocido el valor de RC calculado para cada decisor, se debe verificar que este valor cumpla con lo establecido por el método AHP, valor que está en función del tamaño de la matriz de comparación pareada que para el caso de los criterios $(\mathrm{n}=7)$ no debe superar el $10 \%$ (Tabla 3 ).

Tabla 3. Valores máximos de RC [32]

\begin{tabular}{cc}
\hline $\begin{array}{c}\text { Tamaño de la matriz } \\
\text { de comparación } \\
\text { pareada }(\mathbf{n})\end{array}$ & $\begin{array}{c}\text { Ratio de } \\
\text { consistencia } \\
(\mathbf{R C})\end{array}$ \\
\hline 3 & $5 \%$ \\
4 & $9 \%$ \\
5 o mayor & $10 \%$ \\
\hline
\end{tabular}

\subsubsection{Determinación del factor de ponderación de los criterios y subcriterios de evaluación}

Primero se calcularon los vectores propios empleando el método de la potencia, multiplicando en primera instancia la matriz de comparación pareada por sí misma $A^{2}$ para luego obtener un vector columna que resulta de sumar las filas de la matriz resultante del primer producto, luego se normaliza este vector por medio de la suma y se obtiene el primer vector propio $V_{p 1}$, se siguió el mismo procedimiento para calcular el número de vectores propios necesarios hasta que al comparar las componentes del $V_{p n}$, con $V_{p n-1}$ se verifique que sus componentes correspondientes sean iguales en sus cuatro cifras decimales.

Debido a que fueron consultados tres grupos de interés se empleó la media geométrica [23] para determinar la ponderación final de criterios y subcriterios, consolidando las ponderaciones individuales primero por cada decisor según el grupo y luego por grupo de interés.

\subsection{Tercera fase}

El desarrollo del instrumento de evaluación según propone Quesada [33], considera aspectos como:

- Descripción del criterio de evaluación

- Objetivo de evaluación del criterio

- Método de evaluación

- Tipo de evaluación

- Niveles de referencia

- Exigencias del criterio

\section{- Puntuación y valoración}

En función del instrumento de evaluación se determinaron cuatro niveles de referencia cualitativamente identificados como: insuficiente, bueno, muy bueno y excelente; correspondiéndoles una valoración de 0 , 0.35, 0.7 y 1 respectivamente. La valoración cuantitativa permitió definir el umbral de desempeño al cual está vinculada la valoración cualitativa que se muestra en la Tabla 4.

\subsection{Cuarta fase}

Se realizó la validación del método de evaluación, mediante la aplicación del instrumento desarrollado a cuatro hospitales del IESS de la zona de planificación 3 del Ecuador, los hospitales están ubicados en las capitales provinciales, es decir, en las ciudades de Latacunga, Ambato, Riobamba y Puyo; el muestreo fue intencional debido a que uno de los hospitales de la Zona 3 presentó el más bajo desempeño en la acreditación ACI [17].

Tabla 4. Escala de valoración del desempeño

\begin{tabular}{|c|c|c|}
\hline \multicolumn{2}{|c|}{ Valoración } & \multirow{2}{*}{ Descripción } \\
\hline Cualitativa & Cuantitativa & \\
\hline Insuficiente & {$[0 \%-35 \%)$} & $\begin{array}{l}\text { Presenta deficiencias es- } \\
\text { tructurales que com- } \\
\text { prometen el logro de } \\
\text { los objetivos del depar- } \\
\text { tamento de manteni- } \\
\text { miento. }\end{array}$ \\
\hline Bueno & {$[35 \%-70 \%)$} & $\begin{array}{l}\text { Presenta debilidades es- } \\
\text { tructurales que compro- } \\
\text { meten el logro de los } \\
\text { objetivos, pero existen } \\
\text { procesos viables que } \\
\text { pueden ser considera- } \\
\text { dos para mejorar. }\end{array}$ \\
\hline Muy bueno & {$[70 \%-100 \%)$} & $\begin{array}{l}\text { Presenta debilidades } \\
\text { no estructurales que } \\
\text { pueden solventarse a } \\
\text { través de la mejora de } \\
\text { los procesos puestos en } \\
\text { marcha. }\end{array}$ \\
\hline Excelente & $100 \%$ & $\begin{array}{l}\text { Cumple con las exigen- } \\
\text { cias que requiere este } \\
\text { método de evaluación. }\end{array}$ \\
\hline
\end{tabular}

Los hospitales de Riobamba y Ambato están clasificados como hospitales generales; mientras que los de Latacunga y Puyo como básicos, el número promedio de camas es de 30, todos cuentan con departamento de mantenimiento. La evaluación se realizó en sitio basándose en la verificación física de evidencias [21] que permitan corroborar las afirmaciones del encuestado. 


\subsection{Quinta fase}

Se realizó la identificación de los aspectos de menor desempeño empleando la técnica del diagrama de Pareto; que permitió identificar las potencialidades para mejorar la valoración obtenida.

\section{Resultados y discusión}

\subsection{Resultados}

Como resultados de la primera y segunda fase, y previo a la ponderación de criterios, se verificó que cada valor de RC (Tabla 5) no exceda el límite máximo (10\%).

Tabla 5. Valores de RC (\%) de decisores por grupo de interés

\begin{tabular}{cccc}
\hline N. & $\begin{array}{c}\text { Docentes carrera } \\
\text { de Ingeniería de } \\
\text { Mantenimiento }\end{array}$ & $\begin{array}{c}\text { Especialistas } \\
\text { en } \\
\text { mantenimiento }\end{array}$ & $\begin{array}{c}\text { Administradores } \\
\text { mentenimiento }\end{array}$ \\
\hline 1 & 8 & 9,64 & 7,42 \\
2 & 9,87 & 9,23 & 9,82 \\
3 & 8,92 & 7,37 & 9,95 \\
4 & 8,62 & 8,72 & 8,31 \\
5 & 9,43 & 9,85 & 7,39 \\
6 & 3,24 & 9,64 & 9,66 \\
7 & 7,16 & 8,27 & 5,13 \\
8 & 9,38 & 8,95 & 7,65 \\
9 & 7,64 & 9,83 & 9,36 \\
10 & 9,69 & 6,28 & 9,43 \\
\hline
\end{tabular}

Luego de haber verificado la consistencia en la valoración de los decisores, se obtuvieron las ponderaciones para los criterios y subcriterios de evaluación que se muestran en la Tabla 6 .

En la fase tres se desarrolló el instrumento de valoración que está conformado por veinte subcriterios de evaluación, en la Tabla 7 se indica un ejemplo con el subcriterio OM2 y la estructura del formato que se adoptó para todos los subcriterios, siendo los niveles de exigencia los que varían según cada subcriterio.

Empleando el instrumento de evaluación y definido el umbral de desempeño se procedió con las evaluaciones a los departamentos de mantenimiento de los cuatro hospitales del IESS - Zona 3 cuyos resultados obtenidos se observan en la Figura 2.

En la Tabla 8 se indican las valoraciones globales cuantitativas y cualitativas del desempeño alcanzado por los hospitales, mientras que en la Figura 3 se presentan los subcriterios que deben mejorarse en cada hospital, para cada caso se indica el puntaje que se puede alcanzar si se mejora el subcriterio indicado.

Una vez que se han identificado los criterios con bajo desempeño es necesario establecer un orden de prioridades para implantar un plan de acciones de mejora, indicando los criterios sobre los cuales se debe actuar primero para conseguir el $80 \%$ de los puntos perdidos en la evaluación (Tabla 9).

Tabla 6. Ponderación de criterios y subcriterios de evaluación de la gestión del mantenimiento

\begin{tabular}{|c|c|c|c|}
\hline Criterios & Pesos & $\begin{array}{l}\text { Subcriterios de evalua- } \\
\text { ción }\end{array}$ & Pesos \\
\hline \multirow{3}{*}{ CS } & \multirow{3}{*}{0,25} & $\begin{array}{l}\text { CS1: Política de contrata- } \\
\text { ción }\end{array}$ & 0,48 \\
\hline & & $\begin{array}{l}\text { CS2: Supervisión de los tra- } \\
\text { bajos ejecutados }\end{array}$ & 0,29 \\
\hline & & $\begin{array}{l}\text { CS3: Especificaciones téc- } \\
\text { nicas }\end{array}$ & 0,23 \\
\hline \multirow{3}{*}{$\mathbf{R M}$} & \multirow{3}{*}{0,18} & $\begin{array}{l}\text { RM1: Capacitación y en- } \\
\text { trenamiento }\end{array}$ & 0,43 \\
\hline & & $\begin{array}{l}\text { RM2: Formación profe- } \\
\text { sional }\end{array}$ & 0,42 \\
\hline & & $\begin{array}{l}\text { RM3: Cantidad de per- } \\
\text { sonal de mantenimiento }\end{array}$ & 0,15 \\
\hline \multirow{3}{*}{ MI } & \multirow{3}{*}{0,17} & MI1: Maestro de ítem & 0,46 \\
\hline & & $\begin{array}{l}\text { MI2: Inventario valorado } \\
\text { de ítems }\end{array}$ & 0,28 \\
\hline & & $\begin{array}{l}\text { MI3: Control de existen- } \\
\text { cias }\end{array}$ & 0,26 \\
\hline \multirow{6}{*}{$\mathbf{P P}$} & \multirow{6}{*}{0,13} & $\begin{array}{l}\text { PP1: Indicadores de man- } \\
\text { tenimiento }\end{array}$ & 0,38 \\
\hline & & $\begin{array}{l}\text { PP2: Plan implementado } \\
\text { de mantenimiento preven- } \\
\text { tivo para los equipos médi- } \\
\text { cos }\end{array}$ & 0,18 \\
\hline & & $\begin{array}{l}\text { PP3: Programación de } \\
\text { actividades de manteni- } \\
\text { miento }\end{array}$ & 0,14 \\
\hline & & $\begin{array}{l}\text { PP4: Análisis de criticidad } \\
\text { basado en riesgo }\end{array}$ & 0,12 \\
\hline & & $\begin{array}{l}\text { PP5: Inventario de bienes } \\
\text { a mantener }\end{array}$ & 0,1 \\
\hline & & $\begin{array}{l}\text { PP6: Documentos de man- } \\
\text { tenimiento }\end{array}$ & 0,08 \\
\hline \multirow[t]{2}{*}{ OM } & \multirow[t]{2}{*}{0,11} & $\begin{array}{l}\text { OM1: Políticas de mante- } \\
\text { nimiento }\end{array}$ & 0,78 \\
\hline & & $\begin{array}{l}\text { OM2: Herramienta infor- } \\
\text { mática para la gestión de } \\
\text { mantenimiento }\end{array}$ & 0,22 \\
\hline \multirow[t]{2}{*}{$\mathrm{CE}$} & \multirow[t]{2}{*}{0,1} & $\begin{array}{l}\text { CE1: Presupuesto de man- } \\
\text { tenimiento }\end{array}$ & 0,77 \\
\hline & & $\begin{array}{l}\text { CE2: Porcentaje } \\
\text { cumplimiento de } \\
\text { supuesto ejecutado }\end{array}$ & 0,23 \\
\hline $\mathrm{MC}$ & 0,06 & $\begin{array}{l}\text { MC1: Documentación y } \\
\text { análisis de fallos consecuen- } \\
\text { cias y efectos }\end{array}$ & 1 \\
\hline
\end{tabular}


Tabla 7. Descripción del subcriterio de evaluación «herramienta informática (software) para la gestión de mantenimiento»

\begin{tabular}{|c|c|c|}
\hline \multicolumn{3}{|c|}{$\begin{array}{l}\text { Subcriterio de evaluación: OM2 - Herramienta informática } \\
\text { para la gestión de mantenimiento. }\end{array}$} \\
\hline \multicolumn{3}{|c|}{$\begin{array}{l}\text { Objetivo: Establecer si el hospital posee una herramienta } \\
\text { informática tipo GMAO (Gestión de mantenimiento asistido } \\
\text { por ordenador), CMMS (computerized maintenance manage- } \\
\text { ment system) y EAM (enterprise asset management) para } \\
\text { gestionar la información de la gestión de mantenimiento y el } \\
\text { grado de utilización de la misma. }\end{array}$} \\
\hline \multicolumn{3}{|c|}{$\begin{array}{l}\text { Método de evaluación: Se evalúan las evidencias físicas de } \\
\text { instalación del software y de la operatividad del mismo para } \\
\text { el cumplimiento de la exigencia del criterio. }\end{array}$} \\
\hline $\begin{array}{l}\text { Niveles de } \\
\text { referencia }\end{array}$ & $\begin{array}{l}\text { Exigencias } \\
\text { del criterio }\end{array}$ & Puntuación \\
\hline Insuficiente & $\begin{array}{l}\text { No dispone de CMMS/G- } \\
\text { MAO (Computer mainte- } \\
\text { nance management system) } \\
\text { o dispone de CMMS, pero } \\
\text { no lo utiliza. Evidencia: ve- } \\
\text { rificar que esté instalado el } \\
\text { CMMS. }\end{array}$ & 0 \\
\hline Bueno & $\begin{array}{l}\text { La herramienta informática } \\
\text { para la gestión del mante- } \\
\text { nimiento es una hoja elec- } \\
\text { trónica como Excel o una } \\
\text { base de datos básica como } \\
\text { Access. }\end{array}$ & 0,35 \\
\hline Muy bueno & $\begin{array}{l}\text { Dispone de CMMS y lo usa } \\
\text { para gestionar el manteni- } \\
\text { miento preventivo. Eviden- } \\
\text { cia: plan de mantenimiento } \\
\text { emitido por el CMMS y ór- } \\
\text { denes de trabajo preventivas } \\
\text { emitidas en los últimos } 15 \\
\text { días. }\end{array}$ & 0,7 \\
\hline Excelente & $\begin{array}{l}\text { Dispone de CMMS y lo uti- } \\
\text { liza para calcular los indi- } \\
\text { cadores de gestión de man- } \\
\text { tenimiento. Evidencia: re- } \\
\text { porte de costos, disponibi- } \\
\text { lidad, tiempo medio entre } \\
\text { fallas y tiempo medio para } \\
\text { reparación. }\end{array}$ & 1 \\
\hline
\end{tabular}

Tabla 8. Valoración global del desempeño de los hospitales del IESS - Zona 3

\begin{tabular}{ccc}
\hline $\begin{array}{c}\text { Hospital } \\
\text { del IESS }\end{array}$ & $\begin{array}{c}\text { Valoración } \\
\text { cuantitativa }\end{array}$ & $\begin{array}{c}\text { Valoración } \\
\text { cualitativa }\end{array}$ \\
\hline General Riobamba & 77,8 & Muy bueno \\
Básico de Puyo & 50,9 & Bueno \\
General de Ambato & 50,27 & Bueno \\
Básico de Latacunga & 43,05 & Bueno \\
Promedio de los & & Gestión del \\
hospitales del IESS & 55,5 & mantenimiento \\
de la Zona 3 & & Nivel: Bueno \\
\hline
\end{tabular}

Tabla 9. Orden de criterios sobre los que se debe actuar para mejorar el desempeño

\begin{tabular}{|c|c|}
\hline $\begin{array}{l}\text { Hospital } \\
\text { del IESS }\end{array}$ & $\begin{array}{c}\text { Criterios jerarquizados para recuperar } \\
\text { el } 80 \% \text { de puntos perdidos }\end{array}$ \\
\hline $\begin{array}{c}\text { General } \\
\text { Riobamba }\end{array}$ & $\begin{array}{l}\text { 1. Planificación, programación y control } \\
\text { 2. Mantenimiento correctivo } \\
\text { 3. Recursos humanos }\end{array}$ \\
\hline $\begin{array}{l}\text { Básico } \\
\text { de Puyo }\end{array}$ & $\begin{array}{l}\text { 1. Contratación de servicios de mantenimiento } \\
\text { 2. Planificación, programación y control } \\
\text { 3. Manejo de inventarios de bodega }\end{array}$ \\
\hline $\begin{array}{c}\text { General de } \\
\text { Ambato }\end{array}$ & $\begin{array}{l}\text { 1. Recursos humanos } \\
\text { 2. Manejo de inventarios de bodega } \\
\text { 3. Planificación, programación y control }\end{array}$ \\
\hline $\begin{array}{l}\text { Básico de } \\
\text { Latacunga }\end{array}$ & $\begin{array}{l}\text { 1. Recursos humanos } \\
\text { 2. Contratación de servicios de mantenimiento } \\
\text { 3. Organización del mantenimiento }\end{array}$ \\
\hline
\end{tabular}

\subsection{Discusión}

En la fase de ponderación de los criterios y subcriterios considerados para la evaluación de la gestión del mantenimiento resultó que el criterio «Contratación de los servicios de mantenimiento» es el más importante $(25 \%)$ que debe ser evaluado en la gestión y por lo tanto el que más peso tendrá dentro de la valoración cuantitativa, al considerar que aproximadamente el mantenimiento del $90 \%$ de los equipos de los hospitales es realizado por contratistas externos. Dentro de este criterio, el subcriterio a ser considerado es la «Política de contratación» porque en ella se establecen los criterios de selección del mejor oferente que ejecutará las actividades de mantenimiento en el hospital.

Respecto a los resultados obtenidos en la valoración cuantitativa, el hospital con menor desempeño es el hospital del IESS de Latacunga; en la visita realizada para la aplicación del instrumento y verificación en sitio, se evidenció que no existía un responsable del departamento de mantenimiento designado por la máxima autoridad de la institución y que el último responsable del departamento no tenía formación académica de tercer nivel. Este hecho afectó considerablemente el desempeño del hospital porque el criterio «Recursos humanos» es el segundo más importante en la valoración según este método. Para el caso del hospital del IESS de Latacunga este último es el que tiene más potencial de mejorar $(15,17 \%)$.

Para valorar cuantitativamente la gestión del mantenimiento de los hospitales del IESS de la Zona 3, se optó por calcular el promedio de las valoraciones cuantitativas obtenidas por cada hospital, debido a que cuando los hospitales se sometieron al proceso de certificación fueron evaluados con un mismo instrumento y bajo los mismos criterios de la ACI, sin considerar su categoría; y el instrumento aquí propuesto adoptó los criterios de evaluación de la ACI en lo relacionado al mantenimiento. 
Viscaíno-Cuzco et al. / Evaluación de la gestión del mantenimiento en hospitales del Instituto Ecuatoriano de Seguridad Social de la Zona 3 del Ecuador

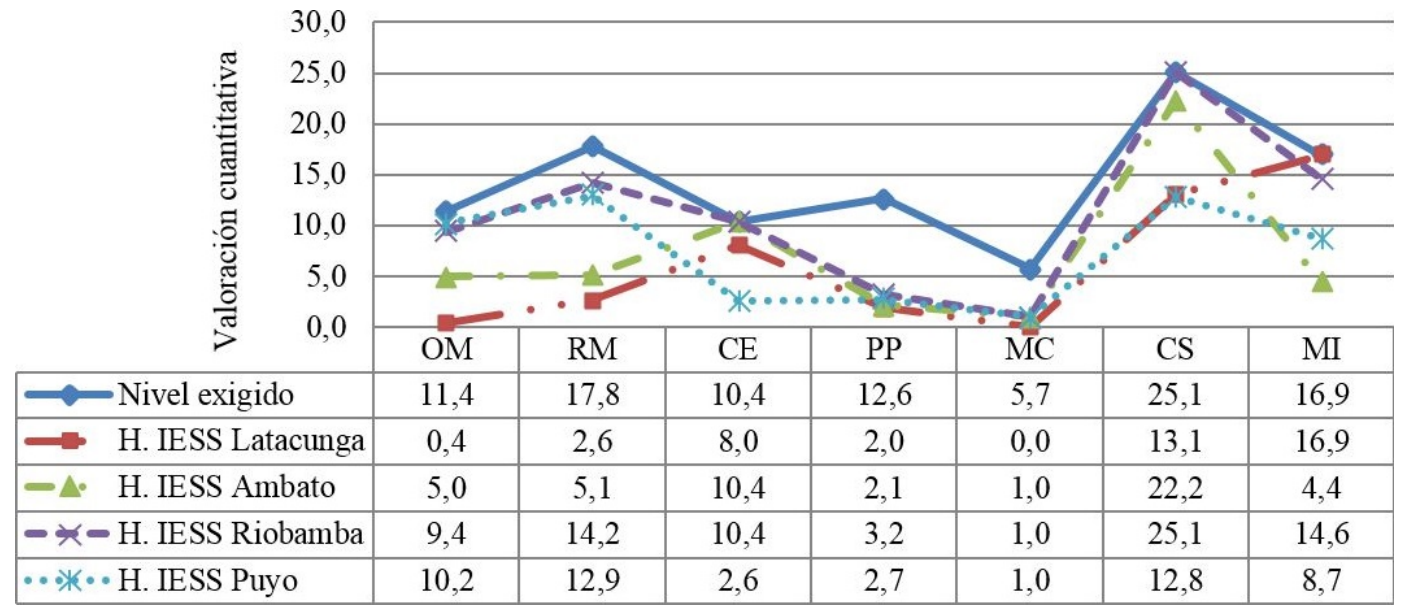

Figura 2. Desempeño de los hospitales del IESS - Zona 3, por criterio de evaluación.

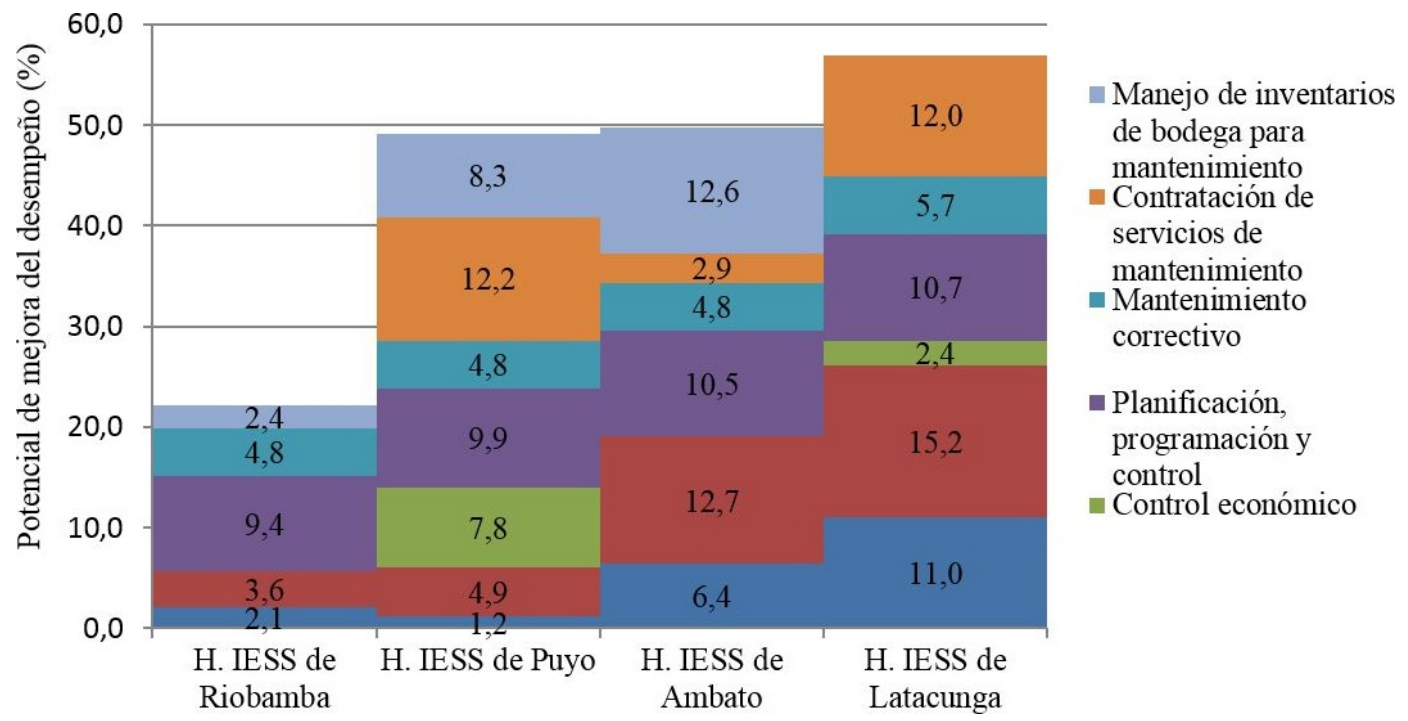

Figura 3. Identificación y potencial de mejora de los criterios con bajo desempeño.

Por lo tanto, en promedio, la gestión del mantenimiento de estos hospitales del IESS es de 55,5 puntos con una desviación estándar de 13 puntos.

Una de las limitaciones que presenta este método es que al momento de la valoración se pueden asignar únicamente los valores establecidos para cada nivel de referencia y no permite dar valoraciones intermedias en el caso de que una de las exigencias se esté cumpliendo parcialmente.

Se considera que este instrumento de evaluación desarrollado puede ser aplicado a otros hospitales, porque todos los centros de salud poseen infraestructura que debe ser mantenida para asegurar una atención de calidad. Además, la Contraloría General del Estado posee un reglamento sobre mantenimiento, que debe ser cumplido [34] también por los hospitales, sin importar su categoría. Esta metodología, no solamente proporciona una valoración cuantitativa, sino que tam- bién indica el orden de criterios sobre los cuales se debe actuar primero para mejorar. Una vez propuestos y puestos en marcha los cambios organizativos conforme a los requerimientos de cada subcriterio, se recomienda realizar anualmente una nueva evaluación a la gestión del mantenimiento aplicando el mismo instrumento de valoración para controlar cuantitativamente el progreso.

\section{Conclusiones}

La encuesta realizada a los especialistas dio como resultado que los tres criterios más importantes son la contratación de servicios de mantenimiento (25\%), recursos humanos (18\%), manejo de inventarios para bodega (17\%); lo que indica que en la evaluación son los criterios que más peso tienen en la valoración. 
Se identificó que ninguno de los hospitales se desempeña según el nivel máximo (nivel deseado) de exigencia del método propuesto, tres de los cuatro hospitales que conforman la Zona 3 presentan un nivel de gestión de mantenimiento en el rango $(35 \%-70 \%)$, que cualitativamente representa una gestión de mantenimiento buena; esto indica que existen debilidades estructurales que comprometen el logro de los objetivos del departamento de mantenimiento, pero hay procesos viables que pueden ser implementados para mejorar.

Existen cuatro criterios en los cuales los cuatro hospitales coinciden que deben mejorar, aunque cada uno en diferente medida y subcriterio, estos son: organización del mantenimiento, recurso humano, planificación, programación y control del mantenimiento y en el mantenimiento correctivo.

\section{Referencias}

[1] H. Alzaben, C. McCollin, and L. Eugene, "Maintenance planning in a saudi arabian hospital," Safety and Reliability, vol. 34, no. 2, pp. 25-40, 2014. [Online]. Available: https: //doi.org/10.1080/09617353.2014.11691004

[2] UNE, UNE-EN 13306:2018. Mantenimiento. Terminología del mantenimiento, Normalización Española Std., 2018. [Online]. Available: http://bit.ly/2RzAv93

[3] V. Gonnelli, F. Satta, F. Frosini, and E. Iadanza, "Evidence-based approach to medical equipment maintenance monitoring," in EMBEC \& $N B C$ 2017, H. Eskola, O. Väisänen, J. Viik, and J. Hyttinen, Eds. Singapore: Springer Singapore, 2018, pp. 258-261. [Online]. Available: https://doi.org/10.1007/978-981-10-5122-7_65

[4] M. C. Carnero and A. Gómez, "A multicriteria decision making approach applied to improving maintenance policies in healthcare organizations," BMC Medical Informatics and Decision Making, vol. 16, no. 1, p. 47, Apr 2016. [Online]. Available: https://doi.org/10.1186/s12911-016-0282-7

[5] R. Lucio, N. Villacrés, and R. Henríquez, "Sistema de salud de Ecuador," Salud Pública de México, vol. 53, pp. s177-s187, 01 2011. [Online]. Available: http://bit.ly/2N2sw5O

[6] INEC, Registro estadístico de recursos y actividades de salud - RAS 2016. relax Instituto Nacional de Estadísticas y Censos, Ecuador, 2016. [Online]. Available: http://bit.ly/2WTPIYL

[7] IESS. (2016) Boletín estadístico número 22. Dirección Actuarial, de Investigación y Estadística del Instituto Ecuatoriano de Seguridad Social.
[8] Asamblea Nacional. (2015) Ley de seguridad social. República del Ecuador. [Online]. Available: http://bit.ly/2ZA0y2Q

[9] P. González, ¿Por qué el IESS se encuentra en crisis? Diario El Comercio, 2019. [Online]. Available: http://bit.ly/2WWxB44

[10] F. Maisanche. (2018) El hospital del IESS de latacunga suspendió las cirugías programadas por problemas en los esterilizadores. Diario El Comercio. [Online]. Available: http://bit.ly/2WTm0Ox

[11] B. G. Mwanza and C. Mbohwa, "An assessment of the effectiveness of equipment maintenance practices in public hospitals," Procedia Manufacturing, vol. 4, pp. 307-314, 2015. [Online]. Available: https://doi.org/10.1016/j.promfg.2015.11.045

[12] A. M. Cruz, G. L. Haugan, and A. M. R. Rincon, "The effects of asset specificity on maintenance financial performance: An empirical application of transaction cost theory to the medical device maintenance field," European Journal of Operational Research, vol. 237, no. 3, pp. 1037-1053, 2014. [Online]. Available: https://doi.org/10.1016/j.ejor.2014.02.040

[13] W. Orozco Murillo, J. Narváez Benjumea, W. García Gómez, and A. Quintero Rodas, "Gestión de mantenimiento y producción más limpia en tres instituciones de salud de Medellín, Colombia," Revista Ingeniería Biomédica, vol. 11, no. 21, pp. 21-25, 2017. [Online]. Available: https: //doi.org/10.24050/19099762.n21.2017.1168

[14] M. Herrera-Galán and E. Martínez-Delgado, "Management audit applied to the maintenance department in hospital facilities," Ingeniería Mecánica, vol. 20, no. 3, pp. 152-159, 2017. [Online]. Available: http://bit.ly/31FcMcp

[15] N. A. A. Rani, M. R. Baharum, A. R. N. Akbar, and A. H. Nawawi, "Perception of maintenance management strategy on healthcare facilities," Procedia - Social and Behavioral Sciences, vol. 170, pp. 272-281, 2015. [Online]. Available: https://doi.org/10.1016/j.sbspro.2015.01.037

[16] L. Thompson, "Acreditación es sinónimo de mejora en atención de salud". Diario El Telégrafo, 2018. [Online]. Available: http://bit.ly/2X6aswb

[17] O. Espín, "Acreditación internacional de hospitales del IESS," in XI Congreso Internacional de Calidad, 2017. [Online]. Available: http://bit.ly/2Y2CAgf

[18] SENPLADES, Plan Estratégico Institucional 2018 - 2021. Coordinación General de Planificación y Gestión Estratégica, Secretaría Nacional 
Viscaíno-Cuzco et al. / Evaluación de la gestión del mantenimiento en hospitales del Instituto Ecuatoriano de

de Planificación y Desarrollo, 2018. [Online]. Available: http://bit.ly/2L38wxh

[19] Acreditation Canada. (2014) Prácticas organizacionales requeridas. Acreditation Canada International, Driving Quality Health Services. [Online]. Available: http://bit.ly/2IufU3j

[20] L. Pecchia and P. Melillo, Multicriteria and Multiagent Decision Making with Applications to Economics and Social Sciences, 2013, vol. 305, ch. Analytic Hierarchy Process for Health Technology Assessment: A Case Study for Selecting a Maintenance Service Contract, pp. 275-288. [Online]. Available: http://bit.ly/2IrWM5K

[21] ISO, ISO 19011: Directrices para la auditoría para sistemas de gestión, International Standard Organization Std Std., 2016. [Online]. Available: http://bit.ly/2XoX8m4

[22] Acosta-Palmer, H. R, and M. Troncoso-Fleitas, "Auditoría integral de mantenimiento en instalaciones hospitalarias, un análisis objetivo," Ingeniería Mecánica, vol. 14, no. 2, pp. 107-118, 2011. [Online]. Available: http://bit.ly/2x1Btld

[23] M. C. Carnero, "Auditing model for the introduction of computerised maintenance management system," International Journal of Data Science, vol. 1, no. 1, pp. 14-41, 2015. [Online]. Available: https://doi.org/10.1504/IJDS.2015.069049

[24] E. Martínez, M. Álvarez, A. Arquero, and M. Romero, "Apoyo a la selección de emplazamientos óptimos de edificios. Localización de un edificio universitario mediante el Proceso Analítico Jerárquico (AHP)," Informes de la construcción, vol. 62, no. 519, pp. 35-45, 2010. [Online]. Available: http://bit.ly/2Y2G3vh

[25] S. Mullally, T. Bbuku, and G. Musonda, "Medical equipment maintenance personnel and training in zambia," in World Congress on Medical Physics and Biomedical Engineering May 26-31, 2012, Beijing, China, M. Long, Ed. Berlin, Heidelberg: Springer Berlin Heidelberg, 2013, pp. 750-753. [Online]. Available: https://doi.org/10.1007/978-3-642-29305-4_197

[26] S. Taghipour, D. Banjevic, and A. K. S. Jardine, "Prioritization of medical equipment for maintenance decisions," Journal of the Operational Research Society, vol. 62, no. 9, pp. 1666-1687, 2011. [Online]. Available: https://doi.org/10.1057/jors.2010.106
[27] M. A. Viscaíno Cuzco, J. F. Quesada Molina, and S. R. Villacrés-Parra, "Priorización de criterios para la evaluación de la gestión del mantenimiento en edificios multifamiliares," Arquitectura y Urbanismo, vol. 38, no. 3, pp. 60-70, 2017. [Online]. Available: http://bit.ly/2FmNeXI

[28] T. L. Saaty, "Decision making with the analytic hierarchy process," International Journal Services Sciences, vol. 1, no. 1, pp. 83-98, 2008. [Online]. Available: http://bit.ly/2IYJEnH

[29] Q. Shen, K.-K. Lo, and Q. Wang, "Priority setting in maintenance management: a modified multiattribute approach using analytic hierarchy process," Construction Management and Economics, vol. 16, no. 6, pp. 693-702, 1998. [Online]. Available: https://doi.org/10.1080/014461998371980

[30] G. Middlehurst, R. Yao, L. Jiang, J. Deng, D. Clements-Croome, and G. Adams, "A preliminary study on post-occupancy evaluation of four office buildings in the UK based on the analytic hierarchy process," Intelligent Buildings International, vol. 10, no. 4, pp. 234-246, 2018. [Online]. Available: https://doi.org/10.1080/17508975.2018.1495607

[31] M. Fouladgar, A. Yazdani-Chamzini, A. Lashgari, E. Zavadskas, and Z. Turskis, "Maintenance strategy selection using ahp and copras under fuzzy environment," International Journal of Strategic Property Management (IJSPM), vol. 16, no. 1, pp. 85-104, 2012. [Online]. Available: https://doi.org/10.3846/1648715X.2012.666657

[32] J. Aznar Bellver and F. Guijarro Martínez, Nuevos métodos de valoración: modelos multicriterio. Universitat Politécnica de Valencia, 2012. [Online]. Available: http://bit.ly/2IrDiOO

[33] F. Quesada Molina, "89/*desarrollo de un método de evaluación de la calidad del ambiente interior para el diseño de viviendas sustentables: Caso de estudio región del bío bío, chile," Revista Hábitat Sustentable, vol. 4, no. 1, pp. 56-67, 2014. [Online]. Available: http://bit.ly/2NeG1Qf

[34] Contraloría General del Estado, Normas de Control Interno de la Contraloría General del Estado. Registro Oficial Suplemento 87 de 14-dic.-2009, República del Ecuador, 2014. [Online]. Available: http://bit.ly/2x3ZtnP 